\title{
SMOOTHING METHOD TO VANISH CORRELATION OF RESIDUAL BY LAGRANGE MULTIPLIERS
}

\author{
K. Takezawa \\ National Institute of Agro-Environmental Sciences \\ Kannondai 3-1-1 Yatabe, Tsukuba-gun, Ibaraki, Japan
}

\begin{abstract}
SUMMARY
A new nonparametric smoothing and interpolation method is suggested to obtain an appropriately smooth curve from noisy data. This is based on the assumptions that neighboring residuals are uncorrelated and that the roughness of estimated value should be as small as possible. The method of Lagrange multipliers is used to solve this conditional minimization problem. It gives linear equations with estimated values as variables. Some examples assured good performance of this procesure concerning both smoothing and interpolation.

Further some applications are possible. First, if the average of the data has to be preserved, another additional condition can be added. Second, the optimal multiplier can be regarded as the degree of smoothness of the data. Third, weighting of the roughness term realizes robustization against outliers.

Almost all smoothing algorithms presented recently, except the exploratory smoothing, attribute the degree of smoothing to the balance between the roughness and the likelihood. However, this new method, called MADONNA (Matrix for Aligning Data with Operationally Nonparametric and Non-correlation-residual Algorithm), do not refer to the likelihood at all. Though this feature seems to be of great importance, its statistical property such as bias is still open.
\end{abstract}

\section{INTRODUCTION}

Many researches have been carried out to draw a smoothed curve from noisy and discrete data. Such techniques are required in wide range of study such as histgram, probability distribution, time-series variation, weather map, distribution map, nonlinear regression, and so on.

The algorithms presented these days are divided into three categories. One is exploratory smoothig(Tucky 1977). Though it is very robust against outliers and the procesure is simple, its statistical basis is not very clear. Another is based on cross validation (G. Wahba and S. Wold 1975, Bowman 1984, Silverman 1985), which has comprehensible justification. However its computational cost is high. The other is a utilization of ABIC(Akaike's Baysian Information Criterion) (Akaike 1980, Yanagimato 1983). It is theoretically clear and has wide range of utilization.

Generally speaking, correlation of residual does not take an important role in these methods, although it is a mean to validate goodness of smoothing. "Trend"(Powell 1970), which yields a criterion for the number of parameters of spline curve fitting, is derived from the expected correlation of neighboring residuals. And the testing for serial correlation in regression has been discussed (Durbin and Watson 1950, 1951, Maddala and Rao 1973).

In thepresent paper, a new nonparametric and interpolation method called MADONNA(Matrix for Aligning Data with Operationally Nonparametric and Noncorrelation-residual Algorithm) is suggested, which attaches great importance to correlation of residuals. And some examples and applications are also shown.

Key Words nonparametric smoothing, nonparametric interpolation, Lagrange multiplier, MADONNA, robust estimation 


\section{Derivation}

The main point of smoothing problem is to reach a compromise between two conflicting requirements:

1. We want remove noise which obscure information of data so that its smoothed curve should be as smooth as possible.

2. However if smoothing is excessive, some important information in data may be lost.

We represent the first requirement as the reduction of the sum of squares of the second derivative of estimated value, which is common to many other methods presented resently.

The originality of the method we will introduce lies in the representation of the second requirement. This condition is treated as the appropriate likelihood of estemated value in most methods suggested so far. However we do not pay attention to the likelihood in this new method. Instead the correlation of neighboring residuals is focused. We assumed that the correlaion of neighboring residuals have to be zero. Though this assumption is suitable only when the number of data is large, it is adoptable without any conditon of the type of probability distribution.

This idea can be regarded as a conditional minimization problems, therefore the method of Lagrange mutipliers can be used to solve this problem, that is,

$$
\begin{gathered}
F\left(\left\{Y_{i}^{*_{i}}\right\}\right)=\sum_{i=2}^{N-1}\left(Y_{i-1}^{*_{i}}-2 Y_{i}^{*}+Y_{i+1}^{*_{i}}\right)^{2}+\lambda \sum_{i=2}^{N} \Delta_{i-1} \Delta_{i}, \\
\frac{\partial F\left(\left\{Y_{i}^{*}\right\}\right)}{\partial Y_{i}^{*}}=0, \frac{\partial F\left(\left\{Y_{i}^{*}\right\}\right)}{\partial \lambda}=0 .
\end{gathered}
$$

where $N=$ the number of data, $\left\{Y_{i}\right\}=$ data, $\left\{Y^{*}{ }_{i}\right\}=$ estimated values,

$$
\lambda=\text { unknown multiplier, } \Delta_{\mathrm{i}}=\mathrm{Y}^{*} \mathrm{i}-\mathrm{Y}_{\mathrm{i}}
$$

The second equation of (1) yields,

$$
\begin{aligned}
& 2 Y_{1}^{*}+(-4+\lambda) Y_{2}^{*}+2 Y_{3}^{*}=\lambda Y_{2} \\
& (-4+\lambda) \mathrm{Y}_{1}^{*}+10 \mathrm{Y}_{2}+(-8+\lambda) \mathrm{Y}_{3}+2 \mathrm{Y}_{4}^{*}=\lambda\left(\mathrm{Y}_{1}+\mathrm{Y}_{3}\right) \\
& 2 Y^{*} \mathrm{i}-2+(\lambda-8) \mathrm{Y}^{*} \mathrm{i}-1+12 \mathrm{Y}^{*} \mathrm{i}+(\lambda-8) \mathrm{Y}^{*} \mathrm{i}+1+2 \mathrm{Y}^{*_{\mathrm{i}}}+2 \\
& =\lambda\left(Y_{i-1}+Y_{i+1}\right) \quad(2 \leqq i \leqq N-1) \\
& 2 Y_{N-3}+(-8+\lambda) Y_{N-2}^{*}+10 Y_{N-1}^{*}+(-4+\lambda) Y_{N}^{*}=\lambda\left(Y_{N-2}+Y_{N}\right) \\
& 2 Y_{N-2}^{*}+(-4+\lambda) Y_{N-1}^{*}+2 Y_{N}^{*}=\lambda Y_{N-1}
\end{aligned}
$$

If (2) is solved with respect to $\mathrm{Y}^{*} \mathrm{i}$, it gives a linear operation of $\mathrm{Y}^{*}{ }_{\mathrm{i}}$. It means that this method can be regarded as a linear filter with a parameter $\lambda$, which yields the degree of smoothing. $\lambda$ have to be determined so as to make the correlation of neiboring residuals zero. Therfore the degree of smoothing can be decided without any help such as cross validation or ABIC.

The opimal $\lambda$ which makes the correlation of neighboring residuals zero cannot be obtained explicitly, so that we have to solve (2) with various $\lambda$ to find optimal one. The optimal $\lambda$ gives optimal $\left\{\mathrm{Y}^{*} \mathrm{i}\right\}$ which is as smooth as possible with condition of null correlation of neighboring residuals. Fortunately since the behavior of the correlatrion with respect to $\lambda$ is simple unless the data 


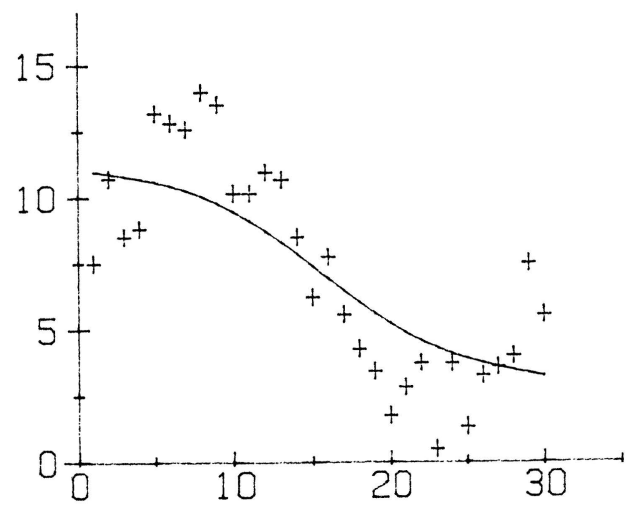

F i g $1 \quad \lambda=3.0, \quad \sum \Delta_{\mathrm{i}} \Delta_{\mathrm{i}-1}=-42.6262$ data $;+$ smoothed value; solid line

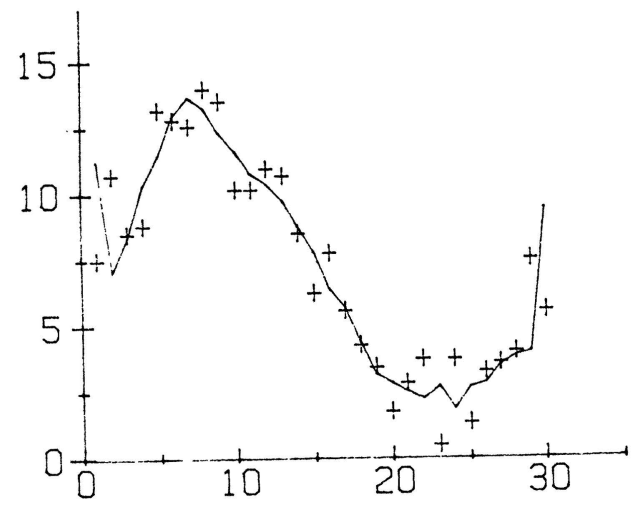

F i g $2 \lambda=0.001, \quad \Sigma \Delta_{\mathrm{i}} \Delta_{\mathrm{i}-1}=86.3983$, data ; + smoothed value ; solid line

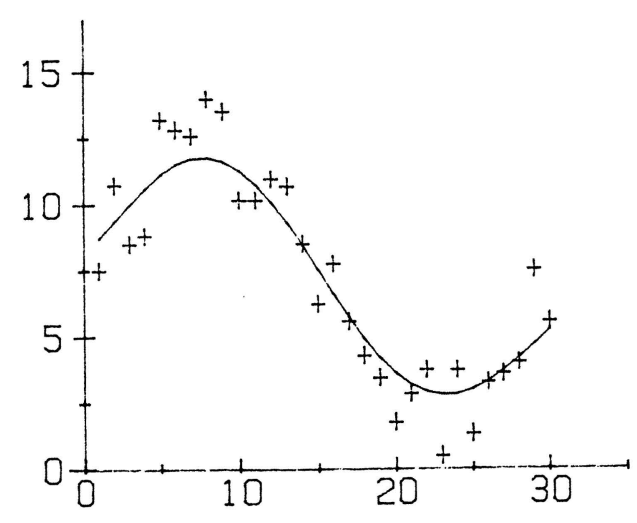

F i g $3 \quad \lambda=0.0123, \quad \sum \Delta_{\mathrm{i}} \Delta_{\mathrm{i}-1}=0.02997$ data ; + smoothed value; solid line

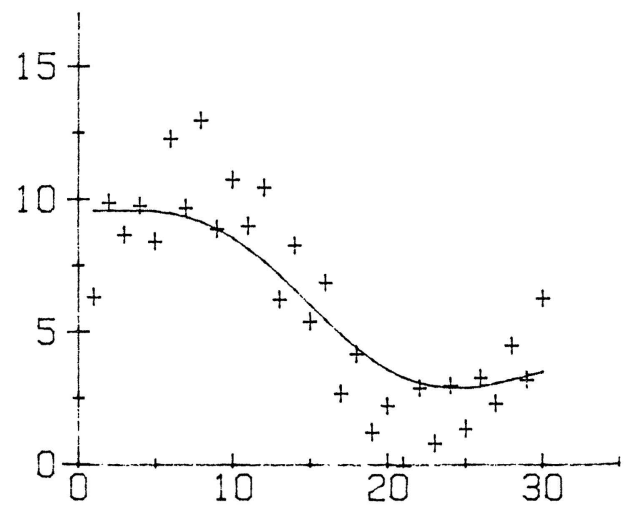

F i g $4 \quad \lambda=0.00275 \quad \Sigma \Delta_{i} \Delta_{i-1}=-0.00199$ data ; + , smoothed value; solid line

is very ill-conditioned, the procesure to find optimal $\lambda$ is not complicated.

To illustrate our algorithm clearly, an example is presented. The data are generated from a sine curve and noisea are added. When $\lambda$ is too small, the estimated values are oversmoothed(Fig 1). When $\lambda$ is too large, the estimated values are undersmoothed(Fig 2). After some calculation to make the correlation of neighboring residual zero, the estimated values become optimal(Fig 3).

A problem occurs when the data is ill-conditioned. For an example, Fig 4, where the neighboring noises has negative correlation, does not show good performance. The estimated values are too smooth in spite of the very small correlation of neighboring residuals. It indicates that if the correlation of noise is clearly correlated, this method can not be applied. We assumed the independence of noise. However, when the noise of data has much correlation by accident, the result can not be adopted. 

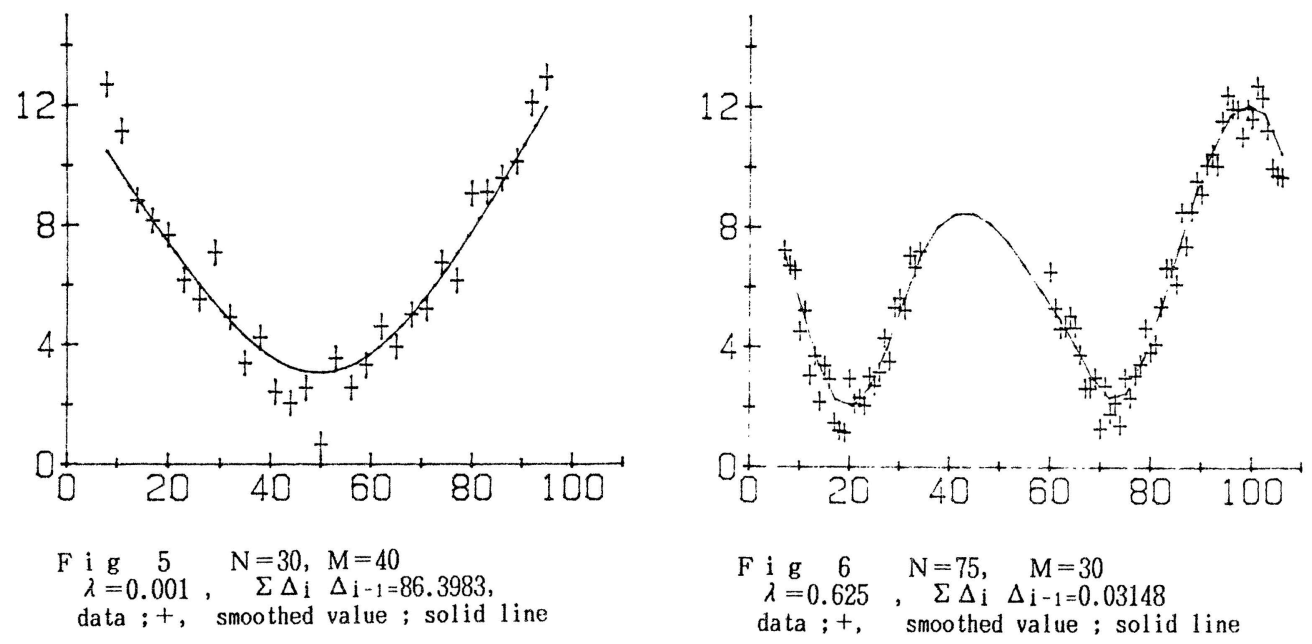

\section{Applications}

\section{1) Interpolation}

The procedure described in section 2. can be used only when the sampling intervals are constant. We have to take a next step to extend the algorithm to interplation, recovering of missing data and making meshed data.

The estimated values taken with equal intervals are linearly interpolated. The equation $\mathrm{F}\left(\left\{\mathrm{Y}^{*} \mathrm{i}\right\}\right)$, becomes,

$$
F\left(\left\{Y^{*}{ }_{i}\right)=\sum_{i=2}^{N-1}\left(Y^{*} i-1-2 Y^{*} i+Y^{*}{ }_{i+1}\right)^{2}+\lambda \sum_{j=1}^{M-1} \sum_{i=1}^{N-1} \varepsilon_{i, j} \Delta_{i}, j \Delta i, j+1,\right.
$$

$\mathrm{M}=$ the number of data, $\mathrm{N}=$ the number of estimated value

$$
\begin{aligned}
& D X=\left(X_{M}-X_{1}\right) /(N-1), \quad X^{*}=X_{1}+D X \times(i-1), \\
& \Delta_{i, j}=Y^{*}+\left(Y^{*}+1-Y_{i}^{*}\right)\left(X j-X^{*}\right) / D X-Y_{j}, \\
& \varepsilon_{i, j}=\left[\begin{array}{ll}
1 & \text { if } X^{*} i \leqq X j<X^{*} i+1 \\
0 & \text { otherwise }
\end{array}\right.
\end{aligned}
$$

If the number of estimated data is taken to be very large, it gives a kind of nonparametric interpolation. Furthermore it can be regarded as recovering missing data or making meshed data. The two examples(Fig 5, Fig 6) shows that intuitively appropriate estimated values are obtained. This method is justified by the fact that the estimatd value whrere the data are missing has zero fourth derivative, that is to say, this interpolation is similar to the spline interpoltion. 2) Conservation of average

If the average of estimated value should be the same as that of the data, a condition can be added to $\mathrm{F}\left(\left\{\mathrm{Y}^{*} \mathrm{i}\right\}\right)$, that is,

$$
\begin{gathered}
F\left(\left\{Y^{*}{ }_{i}\right\}\right)=\sum_{i=2}^{N-1}\left(Y^{*}{ }_{i-1}-2 Y^{*} i+Y^{*}{ }_{i}+1\right)^{2}+\lambda_{1} \sum_{i=2}^{N} \Delta_{i-1} \Delta_{i} \\
\\
\quad+\lambda_{2}\left(\sum_{i=2}^{N-1} Y^{*} /(N-2)-Y\right) .
\end{gathered}
$$




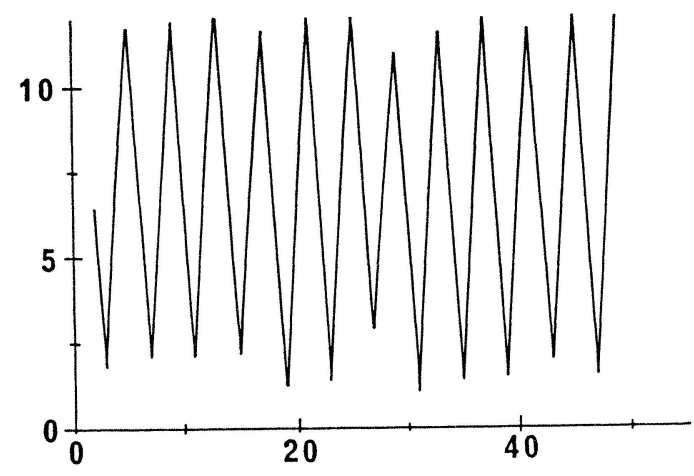

F i g 7 data to calculate $\lambda_{k}$ OPI

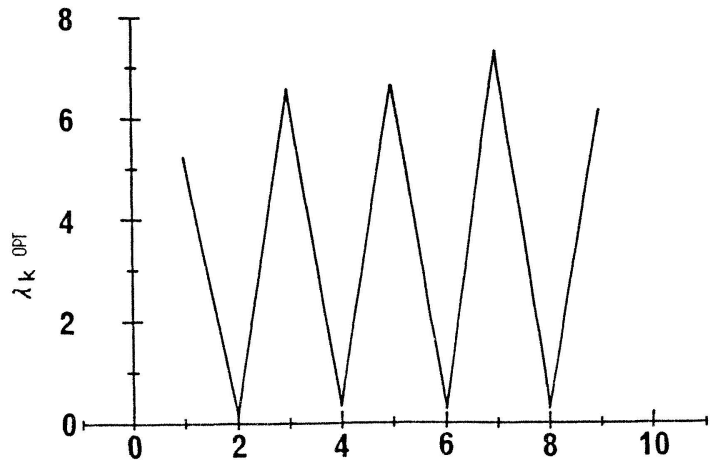

F i g $8\left\{\lambda_{k}\right.$ OPI $\}$ calculated from Fig 7

Conditions can be increased from necessity. However, the estimated value may be unstable if too many conditions are added.

3) $\left\{\lambda_{k}\right.$ OPT $\}$

$\lambda$ can be considered as a kind of ratio between noise and trend. Therefore we define $\lambda_{k}$ OPT as follows.

$$
F\left(\left\{Y_{i}^{*}\right\}\right)=\sum_{i=k+1}^{N-k-1}\left(Y_{i-k}^{*}-2 Y_{i}^{*}+Y^{*} i+k\right)^{2}+\lambda_{k} \sum_{i=k+1}^{N-k} \Delta_{i-k} \Delta_{i} .
$$

$\lambda_{k}$ OPT is determined to make the second term zero for each $k$. It seems that $\lambda_{k}$ OPI represents "Smoothness ratio" in each scale. Figs 7,8 show a simple example of $\left\{\lambda_{k}\right.$ OPI $\}$

Fourier power spectrum is often used to measure the variation of different scale. However it ignores the phase of sine and cosine so that Fourier spectrum is not an appropriate tool to describe variation in some cases. In other words we can not always imagine the variation adaquately from Fourier spectrum. Though some better representation of variation seems to be possible, it is uncertain whether $\left\{\lambda_{k}\right.$ OPI $\}$ can be a applicant for such purpose. Some examples show that $\left\{\lambda_{k}\right.$ OPI $\}$ is unstable so that it may not have high statistical efficiency.

\section{4) Robustization against outliers}

As is often the case in smoothing, outliers do harm to the whole estimated value such as Fig 9. To face this problem, second term of $F\left(\left\{Y^{*} i\right\}\right)$, is weighted as follows.

$$
\begin{aligned}
& F\left(\left\{Y_{i}^{*}\right\}\right)=\sum_{i=2}^{N-1}\left(Y_{i-1}^{*}-2 Y_{i}^{*}+Y_{i+1}^{*}\right)^{2}+\lambda \sum_{i=2}^{N} W_{i} \Delta_{i-1} \Delta_{i}, \\
& W_{i}=1 / \sum_{k=-1}^{1}\left(Y_{i+k-1}-2 Y_{i+k}+Y_{i+k+1}\right)^{2} .
\end{aligned}
$$




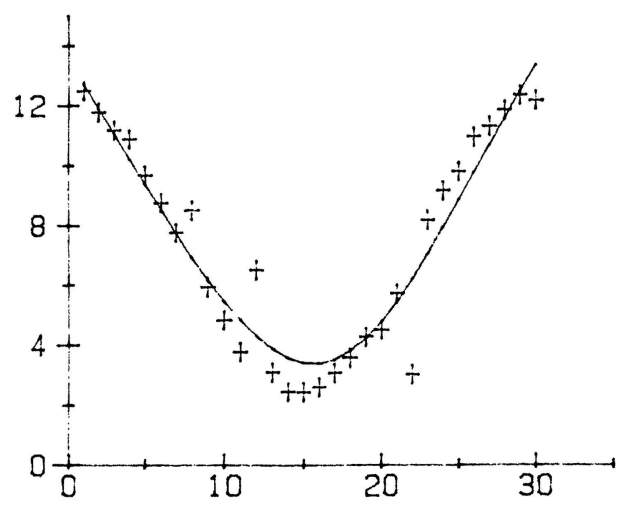

F i g 9 The estimated values by normal MADONNA data ; + , smoothed value; solid line

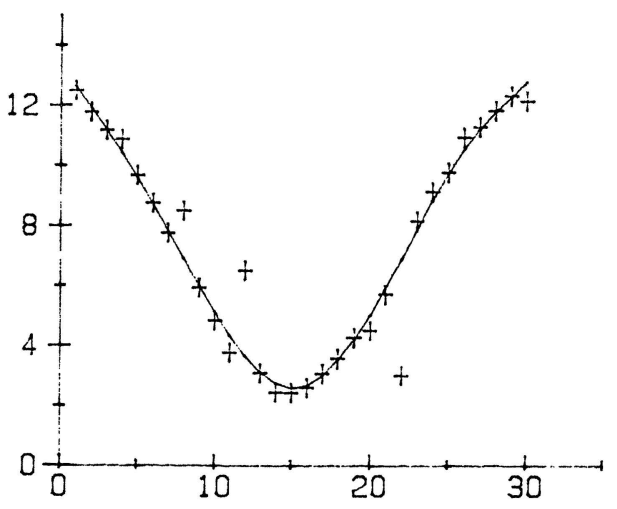

$\mathrm{F}$ i $\mathrm{g} 10$ The estimated values by robustized MADONNA data $;+$ smoothed value; solid line

Robustized estimated values are showm in Fig 10. It is noted that the correlation of neighboring residuals is not zero, but the weighted correlation is zero.

The extent of robustization is a big problem. The robustizations mentioned above is not always appropriate. However, since this method has very simple and coriprehensible structure, it seems not difficult to investigate these problems.

\section{Concluding remarks}

Though this newly ceveloped smoothing and interpolation algorithm has unique features and not so many examples are applied, some points can be said as follows.

(1) In the present mathod, the correlation of neighboring residuals instead of the likelihood is referred and the type of probability distriburtion is not specified. As this is the unique feature of the method, theoretical background is not revealed yet.

(2) The skill of interpolation seems satisfactory. Comparisons to other interpolation methods should be tried to clarify its statistical characteristics such as bias.

(3) Revisions such as robustization or conservation of average are not difficult, because MADONNA has simple and comprehensible structure. It seems that applications to two or three dimensions are not difficult either.

\section{Acknowledgement}

The author is greatly indebted to the helpful comments of Dr. Miwa. The author's thanks also go to Dr. Okada for giving the fascinating nickname, "MADONNA".

\section{References}

Akaike, H.(1980) Likelihood and the Bayes pocedure, Baysian Statistics (eds. J.M.Bernordo, M.H.DeGroot D.V.Lindley and A.F.M.Smith), Univ. Press, Valencia Spain, 143-166.

Bowman, A.W. (1984) An alternative of cross-validation for the smoothing of density estimates Biometrika 71, 353-360

Durbin, J. and Watson, G.S.(1950) Testing for serial correlation in least squares regression 1 Biometrika 37, 409-428.

Durbin, J. and Watson, G.S.(1951) Testing for serial correlation in least squares regression 2 Biometrika 38, 159-178.

Maddala G.S. and Rao A.S.(1973) Test for serial correlation in regression models with lagged dependent variables and serially correlated errors Econometrica 
$41 \quad 761-774$

Powel, M.J.D. Curve fitting by splines in one variable Numerical Approximatrion to Functions and data, J.G.Hayes (ed.), Athlone Press.

Silverman, B.W.(1985). Some aspects of spline smoothing approach to nonparametric regression curve fitting Journal of the Royal Statistical Society B 47, No. 1 1-52.

Tucky, J. W. (1977) Exploratory Data Analysys Section 7 Addison Welsley U.S.A. Wahba, G. and Wold, S. (1975) A completely automatic french curve: fitting spline function by cross validation. Communication in statistics 4(1), 1-17.

Yanagimoyo, T. and Yanagimoto Y.(1983) A procedure to diagnose the goodness of the simple regression model for serial data with application Proceedings of the Institute of Statistical Mathematics Vol 31, No.2 117-127. in Japanese with English summary 